IASSNS-HEP-96/64

hep-ph/9606467

Published: Nucl. Phys. B488 (1997) 141

September 1996

\title{
New Constraints on SO(10) Model-Building from String Theory
}

\author{
Keith R. Dienes" \\ School of Natural Sciences, Institute for Advanced Study \\ Olden Lane, Princeton, N.J. 08540 USA
}

\begin{abstract}
A recent analysis of the methods by which higher-level gauge symmetries are realized in string theory has made it possible to systematically obtain new constraints on string GUT model-building. In this paper, we perform such a study for the case of $S O(10)$ free-field string GUT models, and find a number of significant new results. First, we show that within the conventional string models using the so-called "diagonal" embeddings, all representations larger than the 16 must always transform as singlets under all gauge symmetries beyond $S O(10)$. This includes the $\mathbf{4 5}$ and $\mathbf{5 4}$ representations, and holds regardless of the affine level at which $S O(10)$ is realized. Furthermore, we show that such constructions can never give rise to the 120,126 , or 144 representations of $S O(10)$ - again regardless of the affine level. We also perform a similar analysis for $E_{6}$, and find that the adjoint $\mathbf{7 8}$ representation must always transform as a singlet under all gauge symmetries beyond $E_{6}$; moreover, all representations beyond the $\mathbf{7 8}$ representation are prohibited. Taken together, these results thus severely limit the types of phenomenologically realistic field-theoretic $S O(10)$ and $E_{6}$ models that can be obtained using such string constructions. Finally, we also explore the possibility of using non-diagonal free-field embeddings, and show that in the $S O(10)$ case such embeddings may have improved phenomenological prospects.
\end{abstract}

* E-mail address: dienes@sns.ias.edu 


\section{Introduction}

One of the more popular ways of understanding the apparent unification of gauge couplings within the minimal supersymmetric standard model (MSSM) has been the assumption of an unbroken unified gauge symmetry above the scale of unification. Such a supersymmetric grand-unified theory (GUT) could also in principle explain the quantum numbers of the observed fermion spectrum of the Standard Model, and may ultimately explain the origins of fermion mass. Among the possible GUT models, those utilizing an $S O(10)$ gauge symmetry are particularly compelling [四], since $S O(10)$ is the smallest simple Lie group for which a single anomaly-free irreducible representation can accommodate the entire Standard Model fermion content of each generation. Unification via $S O(10)$ can also provide a natural doublet-triplet splitting mechanism [2], and can accommodate small neutrino masses via the seesaw mechanism [3]. There has therefore been considerable interest in $S O(10) \mathrm{GUT}$ model-building in recent years, and many different proposals currently exist [4, 5, 6].

One way of constraining the set of possible $S O(10)$ unification models is to require that they can be realized as the low-energy limits of heterotic strings. Indeed, in this way, it is hoped that the entire $S O(10)$ framework can be joined with gravity in a consistent string model. For this reason there has been substantial effort in constructing such string GUT models [7, 8, 9, 10], and a review can be found in Ref. [11]. For a long time, the primary difficulty had been the construction of $S O(10)$ models with three generations. Recently, however, there have appeared several promising three-generation $S O(10)$ and $E_{6}$ string GUT models 10. Unfortunately, much of the analysis of these models remains to be done, and in particular these models have not yet been shown to be phenomenologically realistic in other aspects (such as fermion masses, proton lifetime, and so forth). Work along these lines is in progress [10]. Nevertheless, by studying the generic model-independent properties that follow from the string consistency constraints themselves, valuable guidance for low-energy $S O(10)$ model-building can still be obtained. This is the primary goal of the present paper.

Certain types of model-independent information are well-known. For example, in heterotic string models, gauge symmetries are typically realized as affine Lie algebras (also called Kač-Moody algebras) with a given affine level. Thus, simply by studying the unitary representation theory and central charges of $S O(10)$ affine Lie algebras at various levels, one can immediately deduce a number of important constraints. For example, since the central charge of $S O(10)$ at level $k$ is given by $c=45 k /(k+8)$, the perturbative heterotic-string central-charge constraint $c \leq 22$ implies that one can never realize $S O(10)$ at levels $k \geq 8$. Thus, only the cases of $S O(10)$ with levels $1 \leq k \leq 7$ need be considered. This in turn limits the allowed $S O(10)$ representations that can potentially appear in the massless heterotic-string spectrum, and a list of 


\begin{tabular}{c|l|l|l}
$k=1$ & \multicolumn{1}{|c|}{$k=2$} & \multicolumn{1}{|c|}{$k=3$} & \multicolumn{1}{|c}{$k=4$} \\
\hline \hline$(\mathbf{1 0}, 1 / 2)$ & $(\mathbf{1 0}, 9 / 20)$ & $(\mathbf{1 0}, 9 / 22)$ & $(\mathbf{1 0}, 3 / 8)$ \\
$(\mathbf{1 6}, 5 / 8)$ & $(\mathbf{1 6}, 9 / 16)$ & $(\mathbf{1 6}, 45 / 88)$ & $(\mathbf{1 6}, 15 / 32)$ \\
& $(\mathbf{4 5}, 4 / 5)$ & $(\mathbf{4 5}, 8 / 11)$ & $(\mathbf{4 5}, 2 / 3)$ \\
& $(\mathbf{5 4}, 1)$ & $(\mathbf{5 4}, 10 / 11)$ & $(\mathbf{5 4}, 5 / 6)$ \\
& & $(\mathbf{1 2 0}, 21 / 22)$ & $(\mathbf{1 2 0}, 7 / 8)$ \\
& & $(\mathbf{1 4 4}, 85 / 88)$ & $(\mathbf{1 4 4}, 85 / 96)$ \\
& & & $(\mathbf{2 1 0}, 1)$ \\
\hline
\end{tabular}

Table 1: Unitary, potentially massless representations of $S O(10)$ realized at affine levels $k=1,2,3,4$. Each representation $R$ is listed as $\left(\operatorname{dim} \mathbf{R}, h_{R}\right)$ where $h_{R}$ is its conformal dimension. Singlets and conjugate representations are not explicitly written, but understood.

such representations for affine levels $1 \leq k \leq 4$ appears in Table 11.

From this table, a number of additional constraints can be determined. First, it is immediately apparent that the adjoint $\mathbf{4 5}$ representation is possible only in string models in which the $S O(10)$ gauge symmetry is realized at levels $k \geq 2$. Since such adjoint representations are required for the GUT Higgs scalar (in order to break the GUT gauge symmetry down to that of the Standard Model), we see that any consistent $S O(10)$ string GUT model must in fact realize the $S O(10)$ gauge symmetry at $k \geq 2$. Furthermore, if one wishes to obtain a $\mathbf{1 2 0}$ or $\mathbf{1 4 4}$ representation (as is sometimes used for proper light quark/lepton mass ratios as well as for GUT symmetry breaking), one is restricted to levels $k \geq 3$; likewise, in order to achieve a 126 representation (as is often used for a heavy Majorana right-handed neutrino mass, as well as for the Georgi-Jarlskog factor of three in the light quark/lepton mass ratio [12] and for GUT symmetry breaking with automatic $R$-parity conservation), it turns out that one would require $S O(10)$ at levels $k \geq 5$.

Further model-independent constraints can also be gained by studying the conformal dimensions $h_{R}$ of the allowed representations. In string theory, the conformal dimension of a given state describes its spacetime mass (in Planck-mass units), and is related to the number of string excitations needed to produce it. A given state can be massless (i.e., appear in the low-energy string spectrum) if and only if $h_{R} \leq 1$. For example, for $S O(10)$ realized at level $k=2$, the 54 representation has $h=1$. Since this saturates the string masslessness constraint, no additional string excitations are permitted for this representation beyond those that produced the $\mathbf{5 4}$ quantum numbers. In other words, in any level-two $S O(10)$ string model, the 54 representation must be a singlet under all gauge symmetries beyond $S O(10)$. Similar conclusions also hold for the $\mathbf{2 1 0}$ representation of $S O(10)$ at level four. In both cases, such string constraints have profound consequences for the allowed couplings or superpotential 
terms that involve these representations.

The above sorts of results are easy to derive, and have been explored at great length in the literature. For example, a particularly detailed analysis of the constraints following from Table 1 can be found in Ref. [13]. However, all such constraints are based purely on considerations of the affine Lie algebras themselves, such as their central charges and conformal dimensions. Indeed, except for the simple string requirements $c \leq 22$ and $h \leq 1$, they do not rely on any deeper aspects of the methods by which such algebras are ultimately realized within the physical context of string theory. In this paper, however, we shall show that there are additional constraints which arise precisely from such physical considerations in the case of freefield string models. Such free-field string constructions include those based upon free worldsheet bosons and fermions (such as the free-fermionic, lattice, or orbifold constructions [14]), and encompass all of the string constructions which have formed the basis of string GUT model-building attempts in recent years. As shown in Ref. [15], the methods by these higher-level gauge symmetries can actually be realized in such string constructions are highly limited. As a consequence, we shall be able to derive a variety of new constraints which are so powerful that they effectively replace the above table with a new, substantially more restrictive one.

These new methods that we shall use have already proven to be quite powerful when applied to higher-level $S O(10)$ free-field string GUT models. For example, it was recently shown [15] that despite the central charge constraint which naïvely permits $S O(10)$ to be realized at levels $k \leq 7$, in actuality $S O(10)$ cannot be realized beyond level four in such constructions. Thus, Table 1 provides in fact a complete list of potentially allowed representations. This in turn provides the first proof, for example, that the $\mathbf{1 2 6}$ representation of $S O(10)$ can never be realized in free-field heterotic string theory 15 .

In this paper, we shall use the methods of Ref. [15] to obtain a number of further striking results. First, just as the level-two 54 representation transforms as a singlet under all gauge symmetries beyond $S O(10)$, we shall show that in string models employing the so-called "diagonal embeddings" (which includes all string GUT models constructed to date), in fact all representations larger than the $\mathbf{1 6}$ representation must also transform this way. Indeed, we shall show that this result holds regardless of the affine level at which the $S O(10)$ gauge group is realized. In other words, such representations always behave as though they have effective conformal dimensions $h_{\mathrm{eff}}=1$. Second, just as the $\mathbf{1 2 6}$ representation cannot be obtained in free-field string models, we shall show in fact that all representations higher than the 54 cannot be obtained via diagonal embeddings - again, regardless of the level. This includes the useful 120 and 144 representations, which one might have expected to arise at levels $k=3$ or $k=4$. Indeed, the only exception to this rule will be the level-four diagonal embedding, which is capable of yielding a $\mathbf{2 1 0}$ representation. We 
shall also examine the case of $E_{6}$ string models, and prove that similar results hold for this case as well: all adjoint $\mathbf{7 8}$ representations must transform as singlets under all gauge symmetries beyond $E_{6}$, and all representations beyond the $\mathbf{7 8}$ representation are prohibited.

All of these results are completely model-independent, and do not depend on the particular subclass of free-field string model construction that is used (e.g., free fermions, orbifolds, lattices, etc.). Taken together, then, these results place severe restrictions on the types of field-theoretic $S O(10)$ and $E_{6}$ GUT models that can be derived from free-field heterotic string theory using diagonal embeddings. For example, in the case of $S O(10)$, they essentially rule out the models in Ref. [5], and favor the models in Ref. [6]. Given these results, we shall then also explore possible string constructions using non-diagonal embeddings, and show that in the $S O(10)$ case, the phenomenological prospects can be significantly improved.

This paper is organized as follows. In the next section, we shall first review the results of Refs. 115, 16] that will be necessary for our subsequent analysis. Then, in Sect. 3, we will apply these techniques to the case of $S O(10)$ string GUT models built using diagonal embeddings. The case of non-diagonal $S O(10)$ constructions will be considered in Sect. 4, and in Sect. 5 we will turn our attention to $E_{6}$ string GUT models. Finally, in Sect. 6, we will summarize our conclusions and discuss some of their phenomenological ramifications.

\section{Allowed SO(10) Embeddings and Matter Representations}

Let us first begin by briefly reviewing the techniques that underlie our analysis. As we have stated, these techniques are ultimately a consequence of the methods by which free-field string theories yield not only higher-level gauge symmetries, but also their corresponding matter representations. These methods are discussed in Refs. [15, 16] respectively. We shall now review the salient points for each.

\subsection{Allowed SO(10) embeddings}

In Ref. [15], a general examination of the methods by which higher-level gauge symmetries can be realized in string theory was conducted. The main results can be summarized as follows. First, it was shown in Ref. [15] that conformal invariance and the masslessness constraint for gauge boson states together imply that the only way in which a higher-level gauge symmetry $G^{\prime}$ at level $k$ can be realized in freefield heterotic string theory is to start with a certain level-one simply laced gauge symmetry (henceforth denoted $G$ ) with rank $\leq 22$, and then to perform a so-called dimensional truncation of its charge lattice via a GSO projection. Thus, one can realize a higher-level gauge symmetry in free-field string theory only by first building 


\begin{tabular}{|c|c|c|c|c|c|}
\hline Label & \multicolumn{3}{|c|}{ Embedding } & $\Delta r$ & $\Delta c$ \\
\hline 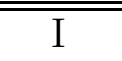 & $S O(10)_{2}$ & $\overline{\bar{C} \subset}$ & $\overline{\left[S O(10)_{1}\right]^{2}}$ & 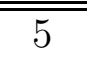 & 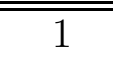 \\
\hline II & $S O(10)_{2}$ & $c$ & $S U(10)_{1}$ & 4 & 0 \\
\hline III & $S O(10)_{3}$ & $c$ & {$\left[S O(10)_{1}\right]^{3}$} & 10 & $30 / 11$ \\
\hline IV & $S O(10)_{4}$ & $\subset$ & {$\left[S O(10)_{1}\right]^{4}$} & 15 & 5 \\
\hline V & $S O(10)_{4}$ & $\subset$ & $S U(16)_{1}$ & 10 & 0 \\
\hline VI & $S O(10)_{4}$ & $\subset$ & {$\left[S U(10)_{1}\right]^{2}$} & 13 & 3 \\
\hline VII & $S O(10)_{4}$ & $\subset$ & $S U(10)_{1} \times\left[S O(10)_{1}\right]^{2}$ & 14 & 4 \\
\hline & $S O(10)_{k>4}$ & & Impossible & & \\
\hline
\end{tabular}

Table 2: Complete list of string-allowed embeddings for $S O(10)$ at higher levels, where $\Delta r \equiv \operatorname{rank} G-5$ and $\Delta c \equiv c(G)-c\left(S O(10)_{k}\right)$. The embeddings have been labelled I through VII for future reference.

a string model which realizes the larger level-one gauge group $G$, and then truncating to the higher-level subgroup $G^{\prime}$. Second, it was also shown [15] that each such dimensional truncation corresponds uniquely to a so-called irregular group-theoretic embedding $G_{k}^{\prime} \subset G$ (where 'irregular' signifies that some of the roots of the subgroup are not roots of the original group). Since these irregular group-theoretic embeddings have been classified by mathematicians, these two observations together permitted a complete classification [15] of the methods by which all higher-level gauge symmetries can be realized in free-field string theory, and the results for the case of $S O(10)$ are given in Table 2. We shall defer our discussion of $E_{6}$ to Sect. 5 .

Note that the embeddings listed in Table 2 are the only ways in which $S O(10)$ at higher levels can be realized in free-field string constructions. Thus, we see that when constructing $S O(10)$ at higher levels, the cost we must pay is not simply that of realizing the $S O(10)$ group factor itself; rather, we must start by actually realizing the larger embedding group $G$, and thereby pay additional costs both in rank $\Delta r$ and in central charge $\Delta c$. When $\Delta c \neq 0$, these extra costs have a profound effect, for they result in the generation of extra unwanted non-gauge chiral algebras of central charge $\Delta c$ in addition to the desired higher-level gauge symmetry [15].

Note that embeddings of the form

$$
S O(10)_{k} \subset\left[S O(10)_{1}\right]^{k}
$$

are the traditional so-called diagonal embeddings. Indeed, most string GUT models constructed to date employ such embeddings: one first realizes the gauge symmetry $\left[S O(10)_{1}\right]^{k}$ in a given string model, and then breaks the gauge symmetry down to the higher-level diagonal subgroup $S O(10)_{k}$ (for example, by modding out by the permutation symmetry between the different $S O(10)$ gauge factors). By contrast, one of the main achievements of Ref. [15] was to identify all of the other non-diagonal 
embeddings which can also produce $S O(10)$ at higher levels. As evident from Table 2, these non-diagonal embeddings are always more efficient than the diagonal embeddings, with smaller $\Delta r$ and $\Delta c$. Indeed, those embeddings with $\Delta c=0$ are called "conformal", and will prove to be extremely useful in what follows since they do not generate any unwanted chiral algebras. Finally, also note that there do not exist any embeddings yielding $S O(10)_{k}$ for $k>4$. Hence such levels cannot be realized in free-field string constructions.

\subsection{Allowed SO(10) matter representations}

While the discussion in Ref. [15] focused on the realization of the $S O(10)$ gauge boson states, in this paper we shall use the results in Table 2 in order to study the possible $S O(10)$ matter representations. In general, just as was the case for the gauge boson states, a given matter representation of $S O(10)$ at any level is permitted if and only if it can also be realized through the embeddings in this table. There are, however, certain subtleties which must be taken into account. In particular, for each embedding $S O(10)_{k} \subset G$, a given matter representation can arise in either an untwisted or a twisted string sector. It turns out that the rules for analyzing the states from the untwisted and twisted sectors may differ depending on the particular embedding $S O(10)_{k} \subset G$ under study. However, as derived and discussed in Ref. [16], there exists a simple rule which governs this process.

In order to discuss this rule, let us first introduce some terminology. We shall say that a string model realizes a given embedding $G_{k}^{\prime} \subset G$ if, as above, this is the embedding through which the gauge bosons of $G_{k}^{\prime}$ are realized. Furthermore, when we label the various sectors of a given string model as either "untwisted" or "twisted", these adjectives will refer to the twist that induced the final breaking $G \supset G_{k}^{\prime}$. Finally, we shall refer to a given matter representation $R^{\prime}$ of any group $G_{k}^{\prime}$ as being "allowed by the embedding" $G_{k}^{\prime} \subset G$ if and only if it arises as the result of the decomposition of a unitary potentially massless representation $R$ of $G$. In other words, there should exist a branching rule of the form $R \rightarrow R^{\prime} \oplus \ldots$. where $R$ is a unitary representation of $G$ with conformal dimension $h \leq 1$.

Given this terminology, the rule for describing the allowed potentially massless matter representations of $S O(10)_{k}$ can be easily summarized as follows 16. If a given model realizes an embedding $S O(10)_{k} \subset G$, then it turns out that a given matter representation of $S O(10)_{k}$ can arise in the corresponding untwisted string sectors only if it is allowed by the same embedding $S O(10)_{k} \subset G$. Moreover, if this embedding $S O(10)_{k} \subset G$ is non-conformal (so that $\Delta c \neq 0$ ), then this restriction must apply to the states from the twisted string sectors as well - i.e., they too must be allowed by the same embedding $S O(10)_{k} \subset G$. Thus, for string models that realize non-conformal embeddings, the states from the twisted and untwisted sectors 
behave similarly, and must arise from decompositions of unitary potentially massless representations of the same original group $G$.

By contrast, if the original gauge-group embedding $S O(10)_{k} \subset G$ is conformal, then it turns out that there is no model-independent constraint that correlates the effective embeddings describing the origins of the twisted-sector matter states with those describing the origins of the untwisted-sector matter states. In other words, while the matter states in the untwisted sectors must still be those that are allowed by the conformal embedding $S O(10)_{k} \subset G$, the matter states from the twisted sectors can in general be those that are allowed by any other embedding $S O(10)_{k} \subset H$ in Table 2 with the same value of $k$. Thus, in general, these twisted-sector states can behave as though they were the untwisted states relative to a different (not necessarily conformal) effective embedding $S O(10)_{k} \subset H$. Note that we are not claiming that such effective embedding "mixtures" will actually arise in a given model — we are merely claiming that they are are allowed in principle. Whether they actually arise depends on the details of the particular string construction.

It is easy to see why the conformal and non-conformal cases are so different. As explained in Ref. [16], the crucial point to consider is the tree-level coupling of the $S O(10)$ gauge bosons to the matter representations that carry $S O(10)$ charges. Such couplings are characterized by the three-point matter/matter/gauge-boson vertices in the low-energy effective field theory. For consistency, such couplings must be non-vanishing regardless of whether these matter representations arise in twisted or untwisted strings sectors. However, this has different implications depending on whether the gauge boson embedding is conformal or non-conformal.

If the gauge boson embedding is non-conformal, then an extra chiral algebra will be generated in addition to the higher-level gauge symmetry. This implies that the full vertex operator for the gauge boson states will contain not only the usual gauge contribution, but also a contribution from the extra chiral algebra. This in turn implies that the gauge bosons cannot couple to charged matter representations unless the vertex operators of such matter states also contain contributions from the same chiral algebra (so that the two contributions may cancel to permit a non-vanishing correlation function). Of course, by definition, this will be the case for matter arising in the untwisted string sectors. However, for matter arising in the twisted string sectors, such couplings cannot exist unless the vertex operators of these states also contain contributions from the same chiral algebra. In other words, it is impossible to couple the gauge bosons to charged twisted-sector matter states unless these twistedsector states also behave as though they are realized via the same embedding. This is ultimately the origin of the rule discussed above.

By contrast, for gauge bosons realized via conformal embeddings, the situation is more flexible because in such cases the vertex operators of the gauge boson states do not carry any contributions from extra chiral algebras. Thus, they can generally 
couple to charged matter representations from all sectors regardless of the particular effective embedding that may be realized in the twisted sectors. Note that this is true even if the effective embedding describing the twisted-sector states is non-conformal. Indeed, in such cases, the twisted-sector matter representations carry their own chiralalgebra contributions, but these nevertheless cancel between themselves in the threepoint matter/matter/gauge-boson vertices. These rules and their ramifications are discussed more fully in Ref. [16].

Thus, for each higher-level $S O(10)$ embedding listed in Table 2, we can now determine the properties of the corresponding potentially massless $S O(10)_{k}$ matter representations simply by determining those that are "allowed" — i.e., by determining those which are realizable via the decompositions of potentially massless representations of the appropriate original groups $G$ according the above rules. This is the procedure we shall follow in this paper.

\section{SO(10): Analysis for Diagonal Embeddings}

To illustrate, let us start by considering the first embedding in Table 2, namely the level-two diagonal embedding $S O(10)_{2} \subset\left[S O(10)_{1}\right]^{2}$. We see from Table 11 that the only allowed potentially massless representations of $S O(10)_{1}$ are the $\mathbf{1 0}$ and $\mathbf{1 6}$ representations, with conformal dimensions $h=1 / 2$ and $5 / 8$ respectively. Thus, the only allowed distinct non-singlet potentially massless representations of $\left[S O(10)_{1}\right]^{2}$ are the $(\mathbf{1}, \mathbf{1 0}),(\mathbf{1}, \mathbf{1 6})$, and $(\mathbf{1 0}, \mathbf{1 0})$ representations, with conformal dimensions $h=$ $1 / 2,5 / 8$, and 1 respectively. Under the diagonal embedding, these representations respectively decompose into the $\mathbf{1 0}, \mathbf{1 6}$, and $\mathbf{1} \oplus \mathbf{4 5} \oplus \mathbf{5 4}$ representations of $S O(10)_{2}$. Thus, in order to realize the $\mathbf{4 5}$ or $\mathbf{5 4}$ representations of $S O(10)_{2}$ via this embedding, we see that we must first realize the $(\mathbf{1 0}, \mathbf{1 0})$ representation of $S O(10)_{1} \times S O(10)_{1}$, and then do the appropriate decomposition.

Note, however, that each of these final representations inherits the conformal dimension of its parent representation. Indeed, the only difference is that after the decomposition, only part of this conformal dimension can be attributed to the $S O(10)_{2}$ gauge affine Lie algebra itself; the remaining contribution arises from the additional chiral algebra. For example, since the original $(\mathbf{1 0}, \mathbf{1 0})$ representation has $h=1$, the final 45 representation that it produces also inherits this conformal dimension: one contribution $h=4 / 5$ arises from the $S O(10)_{2}$ gauge affine algebra itself, and the remaining contribution $h=1 / 5$ arises from the non-gauge chiral algebra. Indeed, $h=1 / 5$ is the extra "cost" of realizing the $\mathbf{4 5}$ representation in this construction. A similar observation for this case appears in Ref. [8]. In fact, since the only way to realize the $\mathbf{4 5}$ representation in this embedding is via the $(\mathbf{1 0}, \mathbf{1 0})$ representation, the 45 representation is correlated with a unique set of excitations of the primary fields of the chiral algebra. This is in fact true of all of the $S O(10)_{2}$ representations 
in this embedding, since each representation has a unique origin in the pre-truncated theory. Thus, for these reasons, we may consider each of the original conformal dimensions to be a true "effective" conformal dimension, so that the 10, 16, 45, and 54 representations of $S O(10)_{2}$ in this embedding have effective conformal dimensions $h_{\mathrm{eff}}=1 / 2,5 / 8,1$, and 1 respectively.

Note that this is a major change beyond what appears in Table $\mathbb{1}$. For example, even though the adjoint $\mathbf{4 5}$ representation at level two has a true conformal dimension $h=4 / 5$, we now see that it nevertheless has an effective conformal dimension $h_{\text {eff }}=1$ in this embedding. Thus, there cannot be any additional excitations that could provide this representation with the quantum numbers of any other gauge groups! Indeed, in this respect, it resembles the $\mathbf{5 4}$ representation which is also a singlet (in this case under the chiral algebra as well as under other gauge groups). Thus, we see that any $\mathbf{4 5}$ or $\mathbf{5 4}$ representations produced via this embedding will be singlets under all other gauge groups.

This result holds for the level-three and level-four diagonal embeddings as well. In the case of the level-three diagonal embedding $S O(10)_{3} \subset\left[S O(10)_{1}\right]^{3}$, the only allowed potentially massless non-singlet representations of $\left[S O(10)_{1}\right]^{3}$ are the $(\mathbf{1}, \mathbf{1}, \mathbf{1 0})$, $(\mathbf{1}, \mathbf{1}, \mathbf{1 6})$, and $(\mathbf{1}, \mathbf{1 0}, \mathbf{1 0})$ representations, along with their permutations. These have conformal dimensions $h=1 / 2,5 / 8$, and 1 respectively, and have branching rules similar to those of the level-two embedding. Thus they too can only give rise to the 10, 16, 45, and 54 representations of $S O(10)_{3}$, with effective conformal dimensions $h_{\mathrm{eff}}=1 / 2,5 / 8,1$, and 1 respectively. The same result also holds for the level-four embedding.

Does this imply that these four representations are the only matter representations that can ever be realized in higher-level $S O(10)$ string models using diagonal embeddings? It may seem that there are two objections. First, as discussed in Sect. 2, it may seem that the above line of reasoning should only apply to the untwisted string sectors. After all, while we are considering the effects of the dimensional truncation on the sectors that were present prior to the twist that induces the dimensional truncation, it may seem that we are ignoring the entirely new twisted sectors that modular invariance forces us to add to the string model. However, any states which arise in the twisted sectors must also obey the same effective diagonal decomposition rules as do the untwisted-sector states. As we have discussed in Sect. 2, this correlation arises because the diagonal embeddings are non-conformal, with $\Delta c \neq 0$. Indeed, as shown in Ref. [16], if the twisted-sector states were to arise in some other manner (e.g., via a different effective embedding $\left.S O(10)_{k} \subset G\right)$, then the gauge bosons from the $S O(10)_{k} \subset\left[S O(10)_{1}\right]^{k}$ embedding could not couple to the charged $S O(10)$ representations from the twisted sectors, for the gauge-boson vertex operators would contain contributions from the $\left[S O(10)_{1}\right]^{k} / S O(10)_{k}$ chiral algebra that would not be cancelled by similar contributions from the vertex operators of the twisted-sector states. 
This failure of the $S O(10)$ gauge bosons to couple to the charged twisted-sector matter states would then signal an inconsistency in the underlying string construction. Thus, the above analysis must hold for both the untwisted and twisted sectors.

The second possible objection to the above results concerns the fact that when studying the decompositions of the allowed representations of $\left[S O(10)_{1}\right]^{k}$, we used the branching rules of the Lie algebra decomposition $S O(10)^{k} \supset S O(10)$ rather than those of the affine Lie algebra decomposition $\left[S O(10)_{1}\right]^{k} \supset S O(10)_{k}$. This is an important distinction, for while the ordinary Lie algebra branching rules are sufficient for understanding the decomposition properties of the finite-dimensional group representations, in our case we actually must deal with the infinite-dimensional affine Lie algebra representations which also include the infinite Virasoro towers of states. Indeed, while the use of the finite branching rules is sufficient for those $S O(10)_{k}$ primary fields that arise from the primary fields of $\left[S O(10)_{1}\right]^{k}$ (and which correspond to the lowest-lying representations in each Virasoro tower of states), it is possible that $S O(10)_{k}$ primary fields can also arise from the descendent fields of $\left[S O(10)_{1}\right]^{k}$ after the $\left[S O(10)_{1}\right]^{k} \supset S O(10)_{k}$ decomposition. This would correspond to the $\left[S O(10)_{1}\right]^{k}$ towers of states reshuffling and recombining to fill out $S O(10)_{k}$ towers of states in such a way that a descendent (excited) state in the original Verma module of the original algebra becomes a primary (lowest-lying) state in the final Verma module. As discussed in Ref. [16], such extra possibilities would be recorded within the full affine branching rules. Thus, in order to investigate this possibility, one must first derive the affine branching rules for the diagonal embeddings $S O(10)_{k} \subset\left[S O(10)_{1}\right]^{k}$.

In general, such affine branching rules are rather difficult to derive, and only partial results exist in the mathematical literature for general embeddings $G^{\prime} \subset G$ [17]. Indeed, these branching rules might seem to be especially complicated in our case since the values of $\Delta c$ that we face are larger than one, and hence the possible chiral conformal field theories whose representations must be tensored with the Verma modules of $G^{\prime}$ have not even been classified.f Fortunately, however, there are two observations that make the job simpler.

First, it is possible to use $S O(10)$ charge conservation in order to quickly constrain the allowed representations that could possibly arise via such descendent fields of $\left[S O(10)_{1}\right]^{k}$. Note that the only potentially massless representations of $\left[S O(10)_{1}\right]^{k}$ that concern us are the $(\mathbf{1}, \mathbf{1}),(\mathbf{1}, \mathbf{1 0}),(\mathbf{1}, \mathbf{1 6})$, and $(\mathbf{1 0}, \mathbf{1 0})$ representations (with a sufficient number of extra singlet representations attached if $k>2$ ). As discussed in Ref. [16], $S O(10)$ charge conservation then implies that the only descendent representations of $S O(10)_{k}$ that could possibly arise from these representations are as follows: 45, 54, and 210 representations from $(1,1)$ and $(\mathbf{1 0}, 10)$; a 120 representation from $(\mathbf{1}, \mathbf{1 0})$; and a $\mathbf{1 4 4}$ representation from $(\mathbf{1}, \mathbf{1 6})$. Of course, after the

* Note that for the level-two diagonal $S O(10)$ embedding, we in fact have $\Delta c=1$. In this case the extra chiral algebra corresponds to that of a boson on a $\mathbf{Z}_{2}$ orbifold of radius $\sqrt{10} / 2$. 
$\left[S O(10)_{1}\right]^{k} \supset S O(10)_{k}$ decomposition, these representations can only appear as primary fields if they are permitted according to Table 1 .

Second, this situation simplifies even further if we now recall that descendent fields have conformal dimensions which always differ by positive integers from those of their underlying primary fields. Thus, we see that the only possible descendent fields that can ever arise in the massless string spectrum must in fact be descendents of the identity representation of $\left[S O(10)_{1}\right]^{k}$, since this is the only representation with $h=0$.

Thus, we reach two very important conclusions. First, we see that the useful 120 and 144 representations of $S O(10)$ never arise as "primary-from-descendent" fields. However, since we have already shown that they also cannot arise as "primary-fromprimary" fields, we learn that they cannot be realized at all! Thus, these representations of $S O(10)_{k}$ are essentially ruled out in diagonal constructions. Note that this result applies regardless of the affine level at which $S O(10)$ is realized. Indeed, we see that the only "large" representation that can ever be realized in such constructions is the $\mathbf{2 1 0}$ representation, which can appear only as a "primary-from-descendent" field at level $k=4$.

Second, we see that although the $\mathbf{4 5}$ and $\mathbf{5 4}$ representations of $S O(10)_{k}$ can now also be realized as "primary-from-descendent" fields, they nevertheless still must transform as singlets under all gauge symmetries beyond $S O(10)$. This is because they can only arise as the first descendents of the identity field, and will therefore continue to have $h_{\text {eff }}=1$. Indeed, this is a general property of all potentially massless "primary-from-descendent" fields, and applies for both diagonal and non-diagonal embeddings. Of course, for conformal embeddings, such fields will not only have $h_{\text {eff }}=1$, but will in fact have $h=1$ (which renders the identification of such candidate representations quite simple).

Thus, to summarize, we have shown the following. For $S O(10)$ diagonal embeddings, we see that the only possible "primary-from-primary" fields that can ever arise are the 10, 16, 45, and $\mathbf{5 4}$ representations, with effective conformal dimensions $h_{\text {eff }}=1 / 2,5 / 8,1$, and 1 respectively. This result holds regardless of the affine level at which $S O(10)$ is realized, and regardless of the particular free-field string construction employed (whether free-fermions, orbifolds, lattice compactifications, etc.). Likewise, the only possible "primary-from-descendent" fields that can ever arise at the massless level are the 45, 54, and 210 representations, but once again we have shown that such representations still must have $h_{\text {eff }}=1$, and must therefore also transform as singlets under all gauge symmetries beyond $S O(10)$. Thus, as a general statement, we see that any representation larger than the $\mathbf{1 6}$ must transform as a singlet under all gauge symmetries beyond $S O(10)$. Moreover, the $\mathbf{1 2 0}$ and 144 representations are excluded in such constructions, regardless of the affine level at which $S O(10)$ is realized. 
Note that while these results hold only for the diagonal embeddings, we see from Table 2 that the diagonal embedding is in fact the only possible embedding in the particular case of $S O(10)$ at level three. Thus, for $S O(10)_{3}$, the above results are in fact completely general. This observation is particularly important, for the only known three-generation $S O(10)$ string GUT models [10] at present are all at level three. As expected, the spectra of these models agree precisely with our general conclusions.

\section{SO(10): Analysis for Non-Diagonal Embeddings}

Given the above results for the diagonal embeddings, let us now turn to the possible non-diagonal embeddings. We shall find that in these cases there is a far richer structure, with many more phenomenologically interesting possibilities.

First, we analyze the non-diagonal level-two embedding, namely $S O(10)_{2} \subset$ $S U(10)_{1}$. In this case, the only allowed potentially massless unitary $S U(10)_{1}$ representations are the $\mathbf{1 0}$ representation with $h=9 / 20$, and the $\mathbf{4 5}$ representation with $h=4 / 5$. These satisfy the $S U(10)_{1} \supset S O(10)_{2}$ branching rules $\mathbf{1 0} \rightarrow \mathbf{1 0}$ and $45 \rightarrow 45$. Thus, this embedding is capable of producing a $\mathbf{1 0}$ representation with $h_{\text {eff }}=9 / 20$, and a 45 representation with $h_{\text {eff }}=4 / 5$. Unlike the diagonal embeddings, this embedding is therefore capable of producing a 45 representation which need not transform as a singlet under all gauge symmetries beyond $S O(10)$.

This embedding, however, can never yield a 16 representation of $S O(10)_{2}$. It may seem that this embedding is therefore useless on phenomenological grounds. Remarkably, however, it turns out that this embedding is conformal, with $\Delta c=0$. This means that it generates no chiral algebra, which in turn means that there is no constraint that forces the twisted-sector states to be realized from the same effective embedding as the untwisted-sector states. In particular, it is possible that the twistedsector states can arise from an effective diagonal embedding, as described above. Thus, it may be possible to build $S O(10)_{2}$ string models in which the $\mathbf{1 6}$ representations with $h_{\mathrm{eff}}=5 / 8$ come from the twisted sectors, while the phenomenologically interesting 45 representations come from the untwisted sectors. Note that this scenario is possible, however, only if the higher-level gauge bosons are realized through this non-diagonal embedding. Also note that the only "primary-from-descendent" representation which can possibly arise in this case is the $\mathbf{5 4}$ representation, since it already has $h=1$. This is because no chiral algebra is present which could supply any missing conformal dimension in order to build $h_{\mathrm{eff}}=1$ for any of the other representations.

Finally, we turn to the non-diagonal embeddings at level four. Considering first the $S O(10)_{4} \subset S U(16)_{1}$ embedding, we see that that the only potentially massless unitary $S U(16)_{1}$ representations are the 16 representation with $h=15 / 32$, and 
the 120 representation with $h=7 / 8$. These satisfy the simple branching rules $\mathbf{1 6} \rightarrow \mathbf{1 6}$ and $\mathbf{1 2 0} \rightarrow \mathbf{1 2 0}$, from which we obtain the $\mathbf{1 6}$ representation of $S O(10)_{4}$ with $h_{\mathrm{eff}}=15 / 32$, and the 120 representation of $S O(10)_{4}$ with $h_{\mathrm{eff}}=7 / 8$. Even though no $S O(10)_{4}$ adjoint or $\mathbf{5 4}$ scalar representation is produced in such untwisted sectors, we need not disregard this embedding because it too is conformal, and thus the states from the twisted sectors may arise as the result of a different effective level-four embedding (such as the level-four diagonal embedding). Also note that, like the above level-two conformal embedding, the only "primary-from-descendent" state that can possibly arise in this case is the $\mathbf{2 1 0}$ state, since it already has $h=1$ at level four.

Next, we consider the $S O(10)_{4} \subset\left[S U(10)_{1}\right]^{2}$ embedding. It is straightforward to see, by the same sort of analysis, that this embedding produces 10, 45, and 54 representations, but no $\mathbf{1 6}$ representation. However, since this embedding is non-conformal, the only possible states from the twisted sectors are the same as those from the untwisted sectors. Thus, unless the necessary $\mathbf{1 6}$ representations can somehow arise as "primary-from-descendent" fields (which is unclear given the fact that the required affine branching rules have not yet been derived in the mathematical literature), we must reject this embedding on phenomenological grounds.

Finally, we consider the $S O(10)_{4} \subset S U(10)_{1} \times\left[S O(10)_{1}\right]^{2}$ embedding. In this case, the only allowed distinct non-singlet potentially massless representations of $S U(10)_{1} \times S O(10)_{1} \times S O(10)_{1}$ are as follows:

$$
\begin{array}{cl}
(\mathbf{1}, \mathbf{1}, \mathbf{1 0}) & \text { with } h=1 / 2 \\
(\mathbf{1}, \mathbf{1}, \mathbf{1 6}) & \text { with } h=5 / 8 \\
(\mathbf{1}, \mathbf{1 0}, \mathbf{1 0}) & \text { with } h=1 \\
(\mathbf{1 0}, \mathbf{1}, \mathbf{1}) & \text { with } h=9 / 20 \\
(\mathbf{4 5}, \mathbf{1}, \mathbf{1}) & \text { with } h=4 / 5 \\
(\mathbf{1 0}, \mathbf{1 0}, \mathbf{1}) & \text { with } h=19 / 20
\end{array}
$$

(along with their permutations). These respectively decompose into the 10; 16; $\{45 \oplus \mathbf{5 4} \oplus \mathbf{1}\} ; \mathbf{1 0} ; \mathbf{4 5}$; and $\{\mathbf{4 5} \oplus \mathbf{5 4} \oplus \mathbf{1}\}$ representations of $S O(10)_{4}$. Thus, we see that this embedding succeeds in providing both of the required 16 and 45 representations. Moreover, we see that the $\mathbf{4 5}$ representation arises with a range of possible effective conformal dimensions depending on how it is produced.

\section{Analysis for $E_{6}$}

Although the above analysis concentrated on the case where the GUT group is $S O(10)$, the methods used are completely general and can thus be applied to any group. In this regard, the case of $E_{6}$ is particularly straightforward, for it has been 
shown [15] that the only possible free-field embeddings yielding $E_{6}$ at higher levels are the diagonal embeddings $\left(E_{6}\right)_{k} \subset\left[\left(E_{6}\right)_{1}\right]^{k}$ for levels $k=2,3$. Indeed, just as it was found that $S O(10)$ cannot be realized beyond level four in free-field constructions [15], it turns out that $E_{6}$ cannot be realized beyond level three. This means, a priori, that the only allowed representations of $E_{6}$ are the $\mathbf{2 7}$ and $\mathbf{7 8}$ representations, with respective conformal dimensions $\{13 / 21,6 / 7\}$ for $k=2$, and $\{26 / 45,4 / 5\}$ for $k=3$. Thus, all representations larger than the $\mathbf{7 8}$ representation of $E_{6}$ are prohibited.

Given the fact that the diagonal embeddings are the only possible embeddings for $E_{6}$, an analysis along the lines presented above is particularly simple. Indeed, we find that among the "primary-from-primary" fields, only the $\mathbf{2 7}$ representation is possible. Such a state arises from the $(\mathbf{1}, \mathbf{2 7})$ or $(\mathbf{1}, \mathbf{1}, \mathbf{2 7})$ representations of $\left[\left(E_{6}\right)_{1}\right]^{k}$, and always has $h_{\mathrm{eff}}=2 / 3$. As in the case of $S O(10)$, this result holds regardless of the level.

At first sight, it may therefore appear that $E_{6}$ string GUT models are ruled out because of the absence of the required adjoint $\mathbf{7 8}$ Higgs representation. However, this is not the case, for such a representation can (and in fact does) appear as a "primary-from-descendent" field. To see that such a representation does appear, let us consider the level-two diagonal embedding, $\left(E_{6}\right)_{2} \subset\left(E_{6}\right)_{1} \times\left(E_{6}\right)_{1}$. This embedding has $\Delta c=6 / 7$, and therefore generates an extra chiral algebra which corresponds to the $m=6$ member of the $c<1$ unitary minimal-model series. This conformal field theory, which is associated with the tricritical three-state Potts model, has primary fields $\phi_{p, q}$ with conformal dimensions $h_{p, q}=\left[(7 p-6 q)^{2}-1\right] / 168$, where $1 \leq p \leq 5$ and $1 \leq q \leq p$. Note that the identity field in this conformal field theory is $\phi_{1,1}$. Under the decomposition

$$
\left(E_{6}\right)_{1} \times\left(E_{6}\right)_{1} \supset\left(E_{6}\right)_{2} \times\{m=3 \text { minimal model }\},
$$

one can then show that the full affine branching rule for the identity representation $(\mathbf{1}, \mathbf{1})$ of $\left(E_{6}\right)_{1} \times\left(E_{6}\right)_{1}$ is

$$
(\mathbf{1}, \mathbf{1}) \rightarrow\left(\mathbf{1}, \phi_{1,1}\right) \oplus\left(\mathbf{7 8}, \phi_{5,5}\right) .
$$

Note that the $\mathbf{7 8}$ representation of $\left(E_{6}\right)_{2}$ has $h=6 / 7$, so that the full $\left(\mathbf{7 8}, \phi_{5,5}\right)$ representation indeed has $h=1$. Thus, as expected, we see that the $\mathbf{7 8}$ primary field of $\left(E_{6}\right)_{2}$ arises as the first descendent of the identity field of $\left(E_{6}\right)_{1} \times\left(E_{6}\right)_{1}$, and has $h_{\text {eff }}=1$. Moreover, we see from this analysis that this is the only way of realizing an adjoint $\mathbf{7 8}$ scalar in $E_{6}$ string GUT models. Conversely, we also see that the $\mathbf{2 7}$ representation of $\left(E_{6}\right)_{2}$ cannot arise this way.

Thus, in the case of $E_{6}$, we have proven that all 27 representations must in fact have $h_{\text {eff }}=2 / 3$; that all $\mathbf{7 8}$ representations must in fact have $h_{\text {eff }}=1$; and that all larger representations are prohibited. This implies, as in the case of $S O(10)$, 
that despite our naïve expectations based on considerations of the affine Lie algebra alone (which would have seemed to permit extra gauge quantum numbers for both the $\mathbf{2 7}$ and $\mathbf{7 8}$ representations), the largest possible representation is in fact the adjoint $\mathbf{7 8}$ representation, and it must always transform as a singlet under all gauge symmetries beyond $E_{6}$. This has indeed been found to be the case in all $E_{6}$ string models constructed to date [9, 10], and seriously restricts the allowed low-energy phenomenologies that such $E_{6}$ string GUT models can have.

Note that similar analyses for $S U(5), S U(6)$, and other GUT groups of interest can also be undertaken along the lines presented here. The results for such cases will be presented elsewhere [16.

\section{Conclusions and Discussion}

The results of our analysis for the case of $S O(10)$ are collected in Table 3, where we have listed the only representations of $S O(10)$ that can actually appear in freefield heterotic string models, along with their "effective" conformal dimensions. Thus, this new Table 3 - rather than the 'standard' Table 1 - is the true reflection of the allowed possibilities for $S O(10)$ string GUT model-building using free-field constructions. The analogous modifications to the 'standard' $E_{6}$ results were discussed in the previous section.

Perhaps the most important difference between Table 1 and Table 3 concerns the increase in the effective conformal dimensions in the latter relative to the conformal dimensions in the former. As we have discussed, this is a reflection of the true "cost" of realizing higher-level gauge symmetries in free-field string models. In particular, note that the $\mathbf{4 5}$ and $\mathbf{5 4}$ representations of $S O(10)$ have $h_{\mathrm{eff}}=1$ for each of the diagonal embeddings. Thus, as we have explained, such 45 and 54 representations must always be singlets under all gauge symmetries beyond $S O(10)$. Also note that despite the appearance of representations larger than 54 in Table 1, we see from Table 3 that such representations are indeed quite difficult to obtain. In fact, other than the $\mathbf{2 1 0}$ representation which appears as a "primary-from-descendent" field at level four, we see that no $S O(10)$ representations larger than the $\mathbf{5 4}$ representation can ever appear for diagonal embeddings, regardless of the affine level. Furthermore, even among the non-diagonal embeddings, such representations appear in only one case: the conformal embedding $S O(10)_{4} \subset S U(16)_{1}$. This is because the "cost" for realizing these representations is pushed too high in all other cases, and consequently they appear only at the Planck scale.

The fact that the diagonal embeddings force the $\mathbf{4 5}$ and $\mathbf{5 4}$ representations to be singlets all gauge symmetries beyond $S O(10)$ has a number of undesirable phenomenological consequences. First, such singlet 45 representations make the doublettriplet splitting mechanism hard to implement. Second, starting from any desired 


\begin{tabular}{|c|c|c|c|c|c|c|}
\hline \multicolumn{2}{|c|}{$k=2$} & $k=3$ & \multicolumn{4}{|c|}{$k=4$} \\
\hline $\mathrm{I}$ & II & III & IV & $\mathrm{V}$ & VI & VII \\
\hline $\begin{array}{l}(\mathbf{1 0}, 1 / 2) \\
(\mathbf{1 6}, 5 / 8) \\
(\mathbf{4 5}, 1) \\
(\mathbf{5 4}, 1)\end{array}$ & $\begin{array}{l}(\mathbf{1 0}, 9 / 20) \\
(\mathbf{4 5}, 4 / 5)\end{array}$ & $\begin{array}{l}(\mathbf{1 0}, 1 / 2) \\
(\mathbf{1 6}, 5 / 8) \\
(\mathbf{4 5}, 1) \\
(\mathbf{5 4}, 1)\end{array}$ & $\begin{array}{l}(\mathbf{1 0}, 1 / 2) \\
(\mathbf{1 6}, 5 / 8) \\
(\mathbf{4 5}, 1) \\
(\mathbf{5 4}, 1)\end{array}$ & $\begin{array}{l}(\mathbf{1 6}, 15 / 32) \\
(\mathbf{1 2 0}, 7 / 8)\end{array}$ & $\begin{array}{l}(\mathbf{1 0}, 9 / 20) \\
(\mathbf{4 5}, 4 / 5) \\
(\mathbf{4 5}, 9 / 10) \\
(\mathbf{5 4}, 9 / 10)\end{array}$ & $\begin{array}{l}(\mathbf{1 0}, 9 / 20) \\
(\mathbf{1 0}, 1 / 2) \\
(\mathbf{1 6}, \mathbf{5} / 8) \\
(\mathbf{4 5}, 4 / 5) \\
(\mathbf{4 5}, 19 / 20) \\
(\mathbf{4 5}, 1) \\
(\mathbf{5 4}, 19 / 20) \\
(\mathbf{5 4}, 1)\end{array}$ \\
\hline
\end{tabular}

Table 3: The "new" complete list of unitary, potentially massless representations of $S O(10)$ which can ever actually appear in free-field heterotic string models. Each representation $R$ is listed as $\left(\mathbf{d i m} \mathbf{R}, h_{\mathrm{eff}}^{(R)}\right)$ where $h_{\mathrm{eff}}^{(R)}$ is its effective conformal dimension. In this table, the embeddings are labelled I through VII as in Table 2. Note that this table does not include possible "primary-from-descendent" fields, which (if present) must always have $h_{\text {eff }}=1$. For Embeddings I and III, no additional "primary-fromdescendent" fields arise; likewise, the only possible "primary-from-descendent" field for Embedding IV is the $\mathbf{2 1 0}$ representation. For all embeddings except II and V, the allowed states from the twisted and untwisted sectors are the same. In the case of Embeddings II and V, only the states allowed in untwisted sectors are shown; the states allowed from twisted sectors can then be any of those listed under Embeddings $\{\mathrm{I}, \mathrm{II}\}$ or $\{\mathrm{IV}, \mathrm{V}, \mathrm{VI}, \mathrm{VII}\}$ respectively.

coupling, they also tend to generate many new unwanted couplings which arise from unconstrained insertions/deletions of $\mathbf{4 5}$ and $\mathbf{5 4}$ singlets. Finally, their singlet nature renders it difficult to construct non-vanishing antisymmetric combinations of multiple $\mathbf{4 5}$ representations, as are often required [18] in order to simulate the effect of the $\mathbf{1 2 6}$ representation usually used for the Georgi-Jarlskog mass relations |12. Indeed, the basic idea here is that an effective $\mathbf{1 2 6}$ representation can be realized in the higherorder superpotential as the totally antisymmetric component of a $\mathbf{4 5} \cdot \mathbf{4 5} \cdot \mathbf{1 0}$ tensor product, but this requires the presence of two 45 representations which must differ in some of their additional quantum numbers in order to permit antisymmetrization.

How then might such antisymmetric couplings be obtained within the diagonal embeddings? From the above analysis, we now see that such antisymmetric couplings can arise only if two or more $\mathbf{4 5}$ representations can be generated which differ either in their right-moving string quantum numbers, or in their quantum numbers under the left-moving chiral algebra. Unfortunately, the first option is generally difficult to arrange (since it turns out to be correlated with the number of generations in the string model), and the second option is not available at all at level $k=2$ [since all $\mathbf{4 5}$ 
representations at level two originate from the same parent $(\mathbf{1 0}, \mathbf{1 0})$ representation of $S O(10)^{2}$. Indeed, this second option is possible only within the $k=3,4$ diagonal embeddings because of the possibility of permuting the factors in the corresponding parent representations $(\mathbf{1}, \mathbf{1 0}, \mathbf{1 0})$ and $(\mathbf{1}, \mathbf{1}, \mathbf{1 0}, \mathbf{1 0})$. In such cases, however, these permutations only respectively yield additional $\mathbf{Z}_{3}$ and $\mathbf{Z}_{6}$ discrete symmetries for the adjoint scalars.

In some sense, these stringent constraints arise for the diagonal embeddings precisely because they are so uneconomical. Indeed, we see from Table 2 that these embeddings are the most expensive, and require the largest possible values of $\Delta r$ and $\Delta c$. It is this which gives rise to the large extra chiral algebras which lead to these strong constraints. By contrast, the non-diagonal embeddings are generally far more efficient, and can yield a greater variety of massless representations with smaller associated costs.

Thus, as model-builders, it seems that we are faced with two options. The first is to continue to use the diagonal embeddings, and to attempt to construct realistic low-energy field-theoretic $S O(10)$ GUT scenarios which can be accommodated within the above constraints. Indeed, the only such field-theoretic $S O(10)$ models which survive are those, such as in Ref. [6], which do not make use of the 120, 126, or 144 representations and which employ few (preferably only one unique) 45 or 54 representation. As pointed out in Ref. [8], however, the scenarios in Ref. [6] may run into serious problems because they require explicit mass terms in the superpotential, and such mass terms cannot arise in general $S O(10)$ string models [8]. The second option, by contrast, is to use the non-diagonal embeddings we have discussed here, for we see that they are not only more economical but also have a far richer structure. In particular, we see that such embeddings appear to have the flexibility to give rise to non-singlet adjoint scalars, as well as possible higher-dimensional representations. Until now, these non-diagonal string embeddings have been little explored, but our results for the case of $S O(10)$ demonstrate that it is perhaps via these embeddings that phenomenologically interesting string GUT models may be found.

\section{Acknowledgments}

I would like to thank S. Barr, G. Cleaver, J. Erler, A. Hanany, C. Kolda, J. Lykken, F. Wilczek, and E. Witten for discussions. I am particularly grateful to K.S. Babu, Z. Kakushadze, E. Kiritsis, and S.-H.H. Tye for many insightful comments throughout the course of this work, and to J. March-Russell for collaborations (Refs. [15, 16]) which supplied many of the general arguments which have been exploited for the $S O(10)$ and $E_{6}$ analyses presented in this paper. I also wish to thank the CERN Theory Group for hospitality while portions of this paper were written. This work was supported in part by DOE Grant No. DE-FG-0290ER40542. 


\section{Note Added}

After this paper was completed, it was pointed out [19] that there is slight loophole in one of the arguments that was used for restricting the matter content arising from the twisted sectors. In certain string models with special patterns of gauge symmetry breaking, the states from twisted sectors cannot necessarily be described as the untwisted states relative to different effective level-one embeddings, and more complicated embedding patterns for such states may emerge. This issue will be discussed fully in Ref. [16]. While no known higher-level chiral string GUT models exhibit this phenomenon (and therefore all known chiral models satisfy the exact constraints we have formulated in this paper), there apparently exists a non-chiral $S O(10)_{3}$ model which makes use of this loophole to yield adjoint representations carrying additional $U(1)$ charges from certain twisted sectors [19]. It is not known whether this loophole can be exploited in realistic (and in particular, chiral) string GUT models. Indeed, this seems rather unlikely, given the results of various searches and classifications of chiral models that have recently been performed. Thus, the technical results of this paper - such as those of Table 3 - are likely to continue to remain valid in realistic string GUT models, while our more general observations that free-field string constructions pose additional constraints on the allowed matter representations in higher-level string models; that "effective" conformal dimensions can be used to rule out the existence of certain large representations that would otherwise be allowed; that diagonal embeddings are very inefficient ways of realizing higher-level gauge symmetries in string theory; and that non-diagonal embeddings may have improved phenomenological prospects - clearly remain valid in any case.

I wish to thank Z. Kakushadze for pointing out the existence of the non-chiral $S O(10)$ model.

\section{References}

[1] H. Georgi, in Particles and Fields (edited by C.E. Carlson), A.I.P. (1975);

H. Fritzsch and P. Minkowski, Ann. Phys. 93 (1975) 193;

A.J. Buras, J. Ellis, M.K. Gaillard, and D.V. Nanopoulos, Nucl. Phys. B135 (1978) 66;

H. Georgi and D.V. Nanopoulos, Nucl. Phys. B155 (1979) 52; Nucl. Phys. B159 (1979) 16;

F. Wilczek and A. Zee, Phys. Rev. D25 (1982) 553.

[2] S. Dimopoulos and F. Wilczek, in Proceedings of Erice Summer School (edited by A. Zichichi), I.T.P. preprint NSF-ITP-82-07 (1981). 
For a recent discussion, see:

K.S. Babu and S.M. Barr, Phys. Rev. D48 (1993) 5354.

[3] M. Gell-Mann, P. Ramond, and R. Slansky, in Supergravity (edited by P. van Nieuwenhuizen and D.Z. Freedman), North-Holland, Amsterdam (1979);

T. Yanagida, in Proceedings of the Workshop on Unified Theories and Baryon Number in the Universe (edited by A. Sawada and A. Sugamoto), K.E.K. preprint 79-18 (1979);

R.N. Mohapatra and G. Senjanovic, Phys. Rev. Lett. 44 (1980) 912.

[4] Some recent $S O(10)$ GUT models can be found in, e.g.,

S. Dimopoulos, L.J. Hall, and S. Raby, Phys. Rev. Lett. 68 (1992) 1984;

H. Arason, D. Castano, E.J. Piard, and P. Ramond, Phys. Rev. D47 (1993) 232;

G. Anderson, S. Raby, S. Dimopoulos, L.J. Hall, and G.D. Starkman, Phys. Rev. D49 (1994) 3660.

[5] X.G. He and S. Meljanac, Phys. Rev. D41 (1990) 1620;

G. Dvali, Phys. Lett. B287 (1992) 101;

D. Lee and R.N. Mohapatra, Phys. Rev. D51 (1995) 1353;

L.J. Hall and S. Raby, Phys. Rev. D51 (1995) 6524.

[6] J. Hisano, H. Murayama, and T. Yanagida, Phys. Rev. D49 (1994) 4966;

K.S. Babu and S.M. Barr, Phys. Rev. D51 (1995) 2463.

[7] D.C. Lewellen, Nucl. Phys. 337 (1990) 61;

A. Font, L.E. Ibáñez, and F. Quevedo, Nucl. Phys. 345 (1990) 389;

G. Aldazabal, A. Font, L.E. Ibáñez, and A.M. Uranga, Nucl. Phys. 452 (1995)

3 ;

S. Chaudhuri, S.-W. Chung, G. Hockney, and J. Lykken, Nucl. Phys. 456 (1995) 89 ;

D. Finnell, Phys. Rev. D53 (1996) 5781;

S. Chaudhuri, G. Hockney, and J. Lykken, Nucl. Phys. B469 (1996) 357;

A.A. Maslikov, I. Naumov, and G.G. Volkov, Int. J. Mod. Phys. A11 (1996) 1117 ;

G. Cleaver, hep-th/9604183.

[8] G. Aldazabal, A. Font, L.E. Ibáñez, and A.M. Uranga, Nucl. Phys. 465 (1996) 34.

[9] J. Erler, Nucl. Phys. B475 (1996) 597.

[10] Z. Kakushadze and S.-H.H. Tye, Phys. Rev. Lett. 77 (1996) 2612; Phys. Rev. D54 (1996) 7520; hep-th/9610106. 
[11] K.R. Dienes, hep-th/9602045 (to appear in Physics Reports).

[12] H. Georgi and C. Jarlskog, Phys. Lett. B86 (1979) 297.

[13] J. Ellis, J. Lopez, and D.V. Nanopoulos, Phys. Lett. B245 (1990) 375.

[14] L.J. Dixon, J. Harvey, C. Vafa, and E. Witten, Nucl. Phys. B261 (1985) 678; Nucl. Phys. B274 (1986) 285;

K. Narain, M. Sarmadi, and C. Vafa, Nucl. Phys. B288 (1987) 551; Nucl. Phys. B356 (1991) 163;

L.E. Ibáñez, J. Mas, H.P. Nilles, and F. Quevedo, Nucl. Phys. B301 (1988) 157;

H. Kawai, D.C. Lewellen, and S.-H.H. Tye, Nucl. Phys. B288 (1987) 1;

I. Antoniadis, C. Bachas, and C. Kounnas, Nucl. Phys. B289 (1987) 87;

H. Kawai, D.C. Lewellen, J.A. Schwartz, and S.-H.H. Tye, Nucl. Phys. B299 (1988) 431.

[15] K.R. Dienes and J. March-Russell, Nucl. Phys. B479 (1996) 113.

[16] K.R. Dienes and J. March-Russell, preprint IASSNS-HEP-96/77 (to appear).

[17] See, e.g., J. Füchs, Affine Lie Algebras and Quantum Groups (Cambridge University Press, Cambridge, England, 1992).

[18] K.S. Babu and R.N. Mohapatra, Phys. Rev. Lett. 74 (1995) 2418.

[19] Z. Kakushadze, private communication. 